\title{
First report of MRI findings in a case of an autoamputated wandering calcified ovary
}

This article was published in the following Dove Press journal:

International Medical Case Reports Journal

17 March 2014

Number of times this article has been viewed

Parag Suresh Mahajan

Nazeer Ahamad

Sheik Akbar Hussain

Department of Radiology, Al-Khor Hospital, Hamad Medical

Corporation, Doha, Qatar

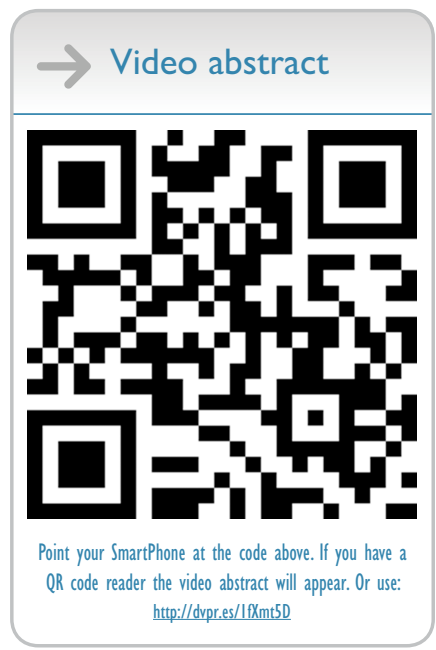

Correspondence: Parag Suresh Mahajan Department of Radiology, Al-Khor Hospital, Hamad Medical Corporation, PO Box 3050, Doha, Qatar

Tel +97466347694

Email mdfrcr@gmail.com
Abstract: An autoamputated wandering calcified ovary (AWCO) is an extremely rare cause of abdominal calcification in the pediatric population. We present the magnetic resonance imaging (MRI) features of AWCO in a child. To our knowledge, the MRI features of AWCO have not been previously described in the published literature. Our case report indicates that the MRI findings are characteristic in the diagnosis of an AWCO and can completely obviate the need for invasive procedures in this mostly benign disease. An AWCO should be considered in all cases of mobile calcific opacities on radiographs in female patients. We advise that MRI be conducted in all suspected cases of AWCO for accurate and noninvasive diagnosis, and regular follow-up should be performed with ultrasound. The findings in our case report have the potential to change the course of investigations and management in suspected cases.

Keywords: magnetic resonance imaging, ovary, adnexa, autoamputation, wandering calcification

\section{Introduction}

Autoamputation of an ovary is an extremely rare condition. ${ }^{1-8}$ Torsion of an ovary or an ovarian lesion and the adnexa can lead to infarction and necrosis and subsequent amputation of the ovary. ${ }^{1-3}$ An amputated ovary gets calcified (dystrophic calcification) and may freely float in the peritoneal cavity. ${ }^{3}$ In most cases, a blunt ending fallopian tube is noted on the involved side with the free-floating calcified ovary during surgery or laparoscopy. ${ }^{3}$ Confirmation of the diagnosis of an autoamputated wandering calcified ovary (AWCO) in most of the previously reported cases was made during surgery or laparoscopy, and all of these cases were evaluated by X-rays, ultrasound, and computed tomography (CT) scan alone or in combination. ${ }^{1-8}$ A magnetic resonance imaging (MRI) study has not been conducted in any of these previously reported cases of AWCO. ${ }^{1-8}$ This is the first MRI report of its kind in the literature of an AWCO in a pediatric patient. ${ }^{1-8}$ We describe the characteristic MRI features of AWCO in this report, and review the literature about the autoamputation of ovaries or the adnexa.

\section{Case material}

A 9-year-old Asian Indian girl presented to the emergency department with a complaint of right lower abdominal pain. The pain was of 2 hours' duration and colicky in nature. She was afebrile and in good general condition. No abdominal guarding, rigidity, or rebound tenderness was noted on examination. Her past medical history was not significant. She was born by normal vaginal delivery in a hospital, and the antenatal and perinatal period was uneventful. Prenatal ultrasound was not done and 
no radiological investigations were done since birth. Urine and blood examinations were normal. A plain erect abdominal X-ray and an abdominal ultrasound were performed. A $1.2 \mathrm{~cm}$ oval calcific opacity was noted in the left side of the pelvis on the abdominal plain X-ray (Figure 1A), and an ultrasound of the abdomen was unremarkable. She was managed conservatively with analgesics. She was sent home after the pain subsided and called again for evaluation of the left pelvic opacity. Another abdominal plain X-ray obtained after a week showed the same opacity in the pelvis in the midline (Figure 1B). A CT scan was performed, suspecting the opacity to be a vesical calculus, which showed an oval calcified lesion in the right adnexa (Figure 2). The CT scan findings raised suspicion of an ovarian dermoid. No fat density was noted in relation to the wandering calcified pelvic lesion. MRI was performed for further evaluation. It showed an absent right ovary and a calcified lesion in the left adnexa adjacent to the left ovary (Figures 3-5), suggesting that it was an autoamputated wandering calcified right ovary. An absent right adnexa and a blunt-ending right fallopian tube were very well visualized on MRI, and they were best visualized on short TI inversion recovery images. A normal left ovary was very well demonstrated on T2-weighted MRI images. On fat suppressed T1-weighted images, the AWCO appeared as an oval hyperintense lesion with a central hypointense area. Peripheral hyperintensity on T1-weighted images in AWCO can be due to methemoglobin or soft calcifications, and the central hypointense area can be due to thick or dense calcifications. The AWCO did not enhance on postcontrast images. Only regular follow-up with ultrasound was advised; no treatment was given, as it is a benign condition and generally does not require surgical

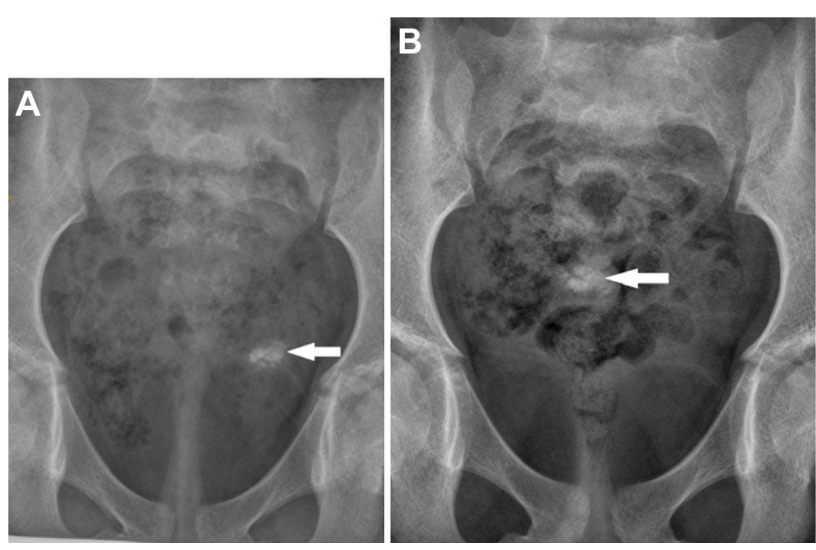

Figure I Abdominal plain X-rays showing an oval calcific opacity.

Notes: Abdominal plain X-rays showing an oval calcific opacity (arrow) (A) in the left pelvic area, (B) which migrated toward the midline in the next abdominal plain X-ray obtained after a week.

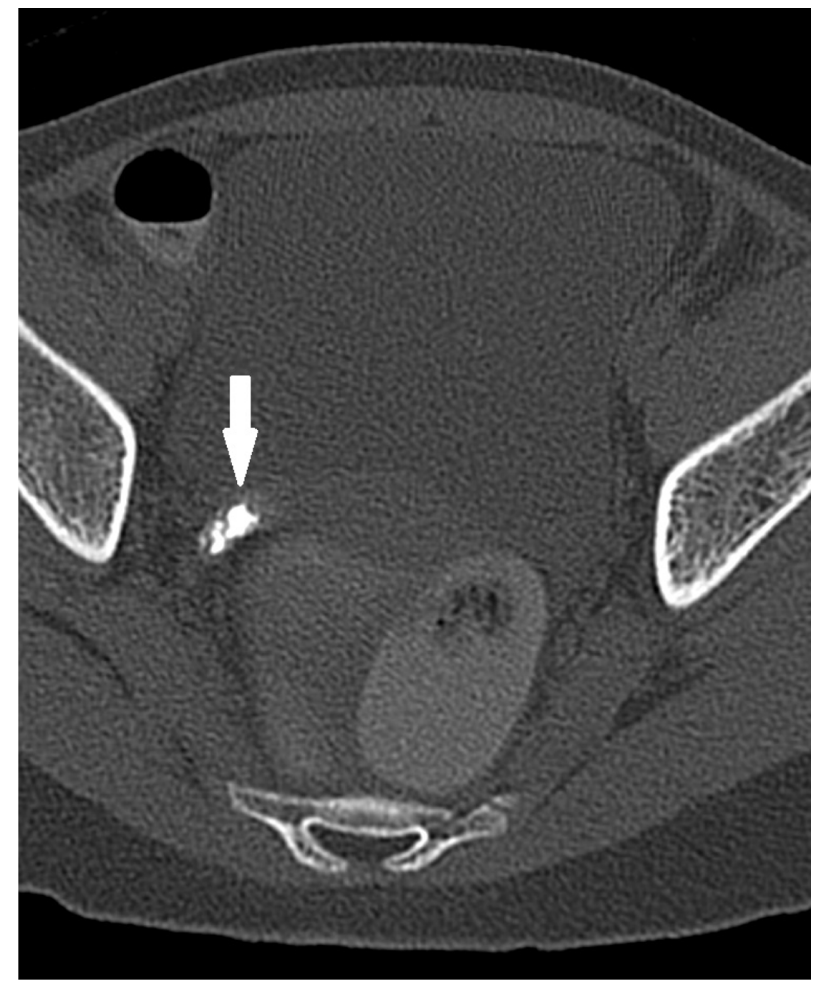

Figure 2 Axial computed tomography scan (bone window) image showing oval calcification (arrow) in the right adnexa.

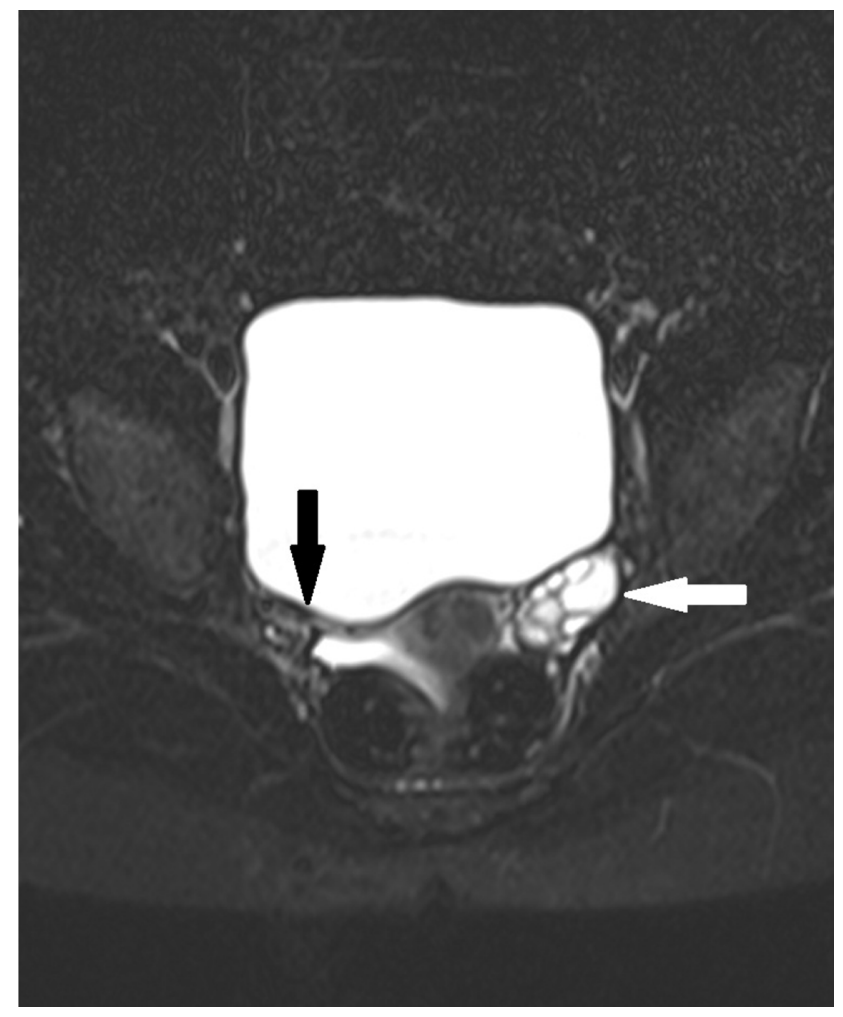

Figure 3 Axial short TI inversion recovery magnetic resonance image showing absent right adnexa and blunt ending right fallopian tube.

Note: Blunt ending right fallopian tube (black arrow); normal left ovary is noted (white arrow). 


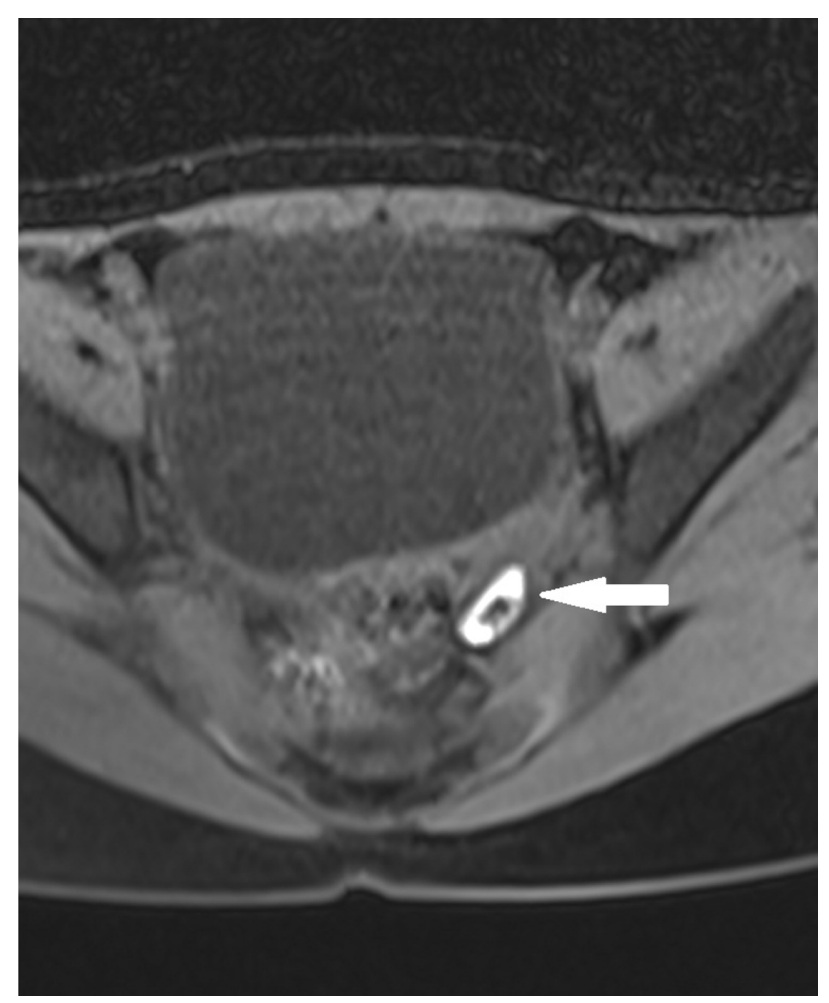

Figure 4 Axial fat suppressed TI-weighted image showing oval hyperintense lesion with thick central calcification in the left adnexa (arrow).

Note: Peripheral hyperintensity on the TI-weighted image can be due to methemoglobin or calcification.

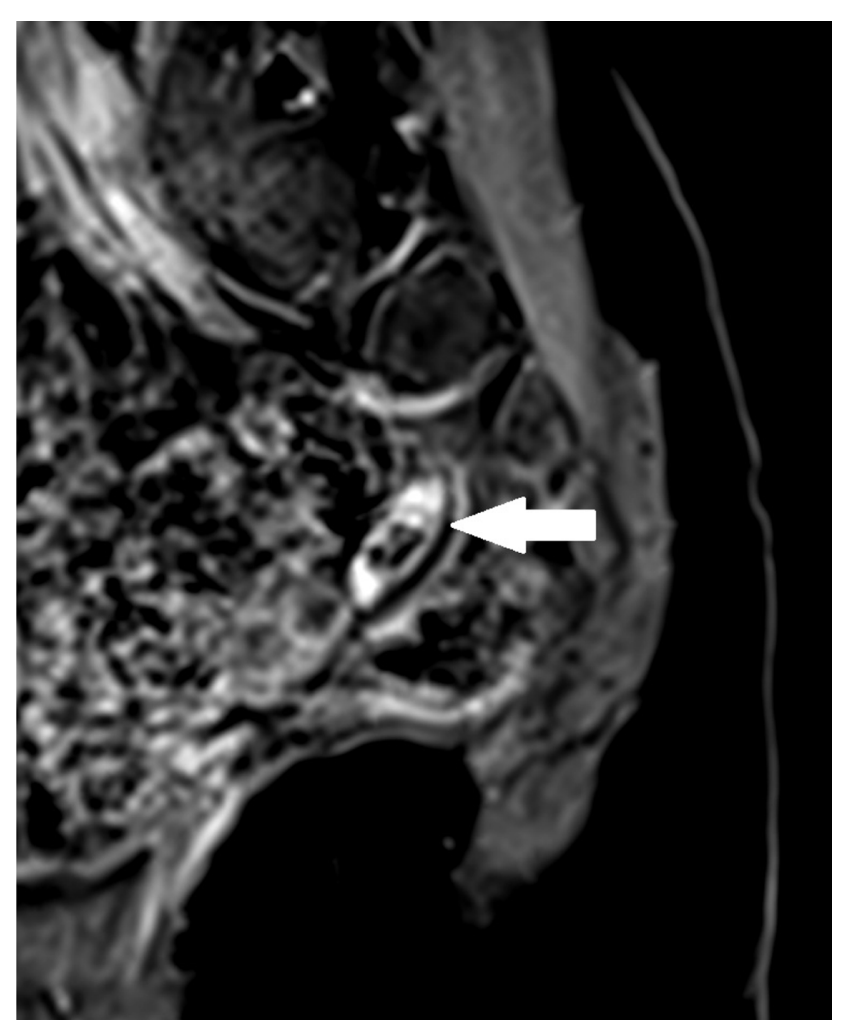

Figure 5 Sagittal post-gadolinium contrast TI-weighted fat suppressed image shows nonenhancing calcified oval lesion (arrow) in the left adnexa. intervention unless complicated. Pain in the abdomen did not recur after the first episode and was considered as unrelated to the AWCO. The patient was followed up for 1 year until the writing of this report with an ultrasound study every 6 months, and no significant changes in the existing findings (or no new findings) were noted. The diagnosis by MRI completely obviated the need for laparoscopy, saving the patient from an unnecessary surgical procedure.

\section{Discussion}

The autoamputation of an ovary is an extremely rare cause of calcification on an abdominal plain X-ray in the pediatric population. ${ }^{3}$ Common causes of the pelvic calcification in young females include teratoma, ovarian neoplasm, chronic ovarian inflammation, appendicolith, urinary bladder calculus, lymph nodal calcification, calcification in a Meckel's diverticulum, gallstone, or neoplasms of the pelvic bones. ${ }^{9}$

Autoamputation of an ovary can be unilateral or bilateral, and it can occur during the prenatal or neonatal period. ${ }^{3}$ It is more common on the right side. ${ }^{1-3}$ The autoamputated ovary is mostly found incidentally while investigating symptoms not related to it, on prenatal ultrasound, or during surgery for different or related causes. ${ }^{1-8}$

In addition to our case, 96 cases of autoamputated ovaries were noted in the published literature, for a total of 97 cases. ${ }^{1-3}$ Half (49/97) of these cases involved pediatric patients ( $<18$ years).$^{1-3}$ Most of these cases were diagnosed via antenatal ultrasonography, likely because it is a very commonly performed examination. ${ }^{3}$ A wandering cystic abdominal mass was demonstrated on antenatal ultrasound in almost all of these cases..$^{1-3}$ In all cases, in older children, the diagnosis was made after laparoscopy or during surgery. ${ }^{1-3}$ Only 14 cases of radiologically demonstrated wandering calcified ovary were identified in the literature, and MRI findings have not yet been described in this group. ${ }^{3}$ In our case, MRI clearly demonstrated the lack of an ovary/adnexa and a freely mobile oval calcified lesion (autoamputated ovary) in the pelvis, suggesting that it was an autoamputated wandering calcified right ovary. The MRI findings are characteristic in the diagnosis of an AWCO. Ultrasonography can show the absence of the adnexa, but MRI is superior in delineating soft tissue morphology. Also, ultrasonography may not demonstrate the displaced calcified ovary. ${ }^{10} \mathrm{CT}$ scans can show calcifications very well, but they are limited in terms of resolving soft tissue abnormalities. ${ }^{9}$ Radiation risk from CT scans can be a concern in young females. ${ }^{11} \mathrm{X}$-rays can only demonstrate mobile calcifications. Therefore, MRI is far better in conclusively demonstrating the absent adnexa and 
also in localizing the wandering calcified ovary, thus making it the investigation of choice in suspected cases of AWCO.

\section{Conclusion}

The MRI findings are characteristic in the diagnosis of an AWCO, and they can completely obviate the need for laparoscopy in this benign condition. An AWCO should be suspected in all cases of mobile calcific opacities on radiographs in females. We advise that MRI be performed in all suspected cases of AWCO for accurate and noninvasive diagnosis, and that regular follow-up with ultrasound be performed. The findings in our case report have the potential to change the management of AWCO in suspected cases.

\section{Disclosure}

The authors report no conflicts of interest in this work.

\section{References}

1. Trotman GE, Zamora M, Gomez-Lobo V. Non-surgical management of the auto-amputated adnexa in the neonate: a report on two cases. J Pediatr Adolesc Gynecol. Epub 2013 Sept 26.
2. Focseneanu MA, Omurtag K, Ratts VS, Merritt DF. The auto-amputated adnexa: a review of findings in a pediatric population. J Pediatr Adolesc Gynecol. 2013;26(6):305-313.

3. Uygun I, Aydogdu B, Okur MH, Otcu S. The first report of an intraperitoneal free-floating mass (an autoamputated ovary) causing an acute abdomen in a child. Case Rep Surg. 2012;2012:615734.

4. Koga K, Hiroi H, Osuga Y, Nagai M, Yano T, Taketani Y. Autoamputated adnexa presents as a peritoneal loose body. Fertil Steril. 2010;93(3): 967-968.

5. Matsushita H, Kurabayashi T, Yanase T, Hashidate H. Autoamputation of an ovarian cyst: a case report. J Reprod Med. 2009;54(11-12):709-711.

6. Peitsidou A, Peitsidis P, Goumalatsos N, Papaspyrou R, Mitropoulou G, Georgoulias N. Diagnosis of an autoamputated ovary with dermoid cyst during a Cesarean section. Fertil Steril. 2009;91(4):1294.e9-e12.

7. Visnjic S, Domljan M, Zupancic B. Two-port laparoscopic management of an autoamputated ovarian cyst in a newborn. J Minim Invasive Gynecol. 2008;15(3):366-369.

8. Kusaka M, Mikuni M. Ectopic ovary: a case of autoamputated ovary with mature cystic teratoma into the cul-de-sac. J Obstet Gynaecol Res. 2007;33(3):368-370.

9. Kaeser MA, McDonald JK, Kettner NW. A calcific pelvic mass in a woman with chronic spinal pain: a case of mature cystic teratoma. J Chiropr Med. 2011;10(4):327-332.

10. McCarthy S. Magnetic resonance imaging in obstetrics and gynecology. Magn Reson Imaging. 1986;4(1):59-66.

11. Berrington de González A, Mahesh M, Kim KP, Bhargavan M, Lewis R, Mettler F, Land C. Projected cancer risks from computed tomographic scans performed in the United States in 2007. Arch Intern Med. 2009; 169(22):2071-2077.
International Medical Case Reports Journal

\section{Publish your work in this journal}

The International Medical Case Reports Journal is an international, peer-reviewed open-access journal publishing original case reports from all medical specialties. Previously unpublished medical posters are also accepted relating to any area of clinical or preclinical science. Submissions should not normally exceed 2,000 words or

\section{Dovepress}

4 published pages including figures, diagrams and references. The manuscript management system is completely online and includes a very quick and fair peer-review system, which is all easy to use. Visit $\mathrm{http}: / / \mathrm{www}$.dovepress.com/testimonials.php to read real quotes from published authors. 